\title{
Enhanced pluggable out-of-plane coupling components for Printed Circuit Board-level optical interconnections
}

\author{
J. Van $\operatorname{Erps}^{a *}$, S. Heyvaert ${ }^{a}$, C. Debaes ${ }^{a}$, B. Van Giel ${ }^{a}$, \\ N. Hendrickx ${ }^{b}$, P. Van Daele ${ }^{b}$, H. Thienpont ${ }^{a}$ \\ ${ }^{a}$ Vrije Universiteit Brussel, Dept. of Applied Physics and Photonics (FirW-TONA), \\ B-1050 Brussel, Belgium \\ ${ }^{b}$ Universiteit Gent, Dept. of Information Technology (INTEC-TFCG), \\ B-9052 Zwijnaarde, Belgium.
}

\begin{abstract}
We present an enhanced out-of-plane coupling component for Printed Circuit Board-level optical interconnections. Rather than using a standard $45^{\circ}$ micro-mirror to turn the light path over $90^{\circ}$, we introduce a curvature in the mirror profile and incorporate an extra cylindrical micro-lens for beam collimation. Both modifications enable an increase in coupling efficiency and are extensively investigated using non-sequential ray tracing simulations in combination with Matlab optimization algorithms. The resulting design is fabricated using Deep Proton Writing and experimental characterization of the geometrical properties and measured coupling efficiencies are presented.
\end{abstract}

Keywords: coupling components, Deep Proton Writing (DPW), micro-mirror, non-sequential ray tracing, optical simulations, polymer waveguides, rapid prototyping

\section{INTRODUCTION}

In the future, the communication bandwidth inside data processing systems will be severely limited by the properties of galvanic interconnections. ${ }^{1}$ These limitations stem from physical constraints imposed by RC time constants and transmission line effects such as frequency dependent loss, crosstalk and reflections. Optics is a potential alternative route to circumvent the underlying problems of galvanic interconnects and is also said to have the potential to continue to scale with future generations of silicon integrated circuits. ${ }^{2}$ Optical interconnects based on low-loss waveguides integrated on a printed circuit board are a promising solution to overcome the interconnect bottlenecks while still relying on standard board technology. ${ }^{3,4}$ However, one of the most critical problems that remains unsolved is the coupling of light in and out of the optical guiding plane. A common approach is the use of $45^{\circ}$ micro-mirrors to realize a $90^{\circ}$ bending of the light. Various techniques are being applied for the fabrication of these micro-mirrors. Micro-machining using a $90^{\circ} \mathrm{V}$-shaped diamond blade ${ }^{5,6}$ can provide an excellent cut surface, but it is difficult to cut individual waveguides on the same substrate due to the physical size of the machining tool. Reactive ion etching ${ }^{7}$ where the slope of the mirror is formed by $45^{\circ}$ oblique etching is limited by directional freedom. Gray-scale lithography ${ }^{8}$ has a very narrow processing window, making it difficult for manufacturing. Other techniques are tilted X-ray exposure ${ }^{9}$ and laser ablation, for which e.g. KrF Excimer lasers are used. ${ }^{10,11}$ All the above technologies are used to write the micro-mirrors directly in the waveguides.

We recently reported a completely different approach, consisting of a discrete out-of-plane coupling component for Printed Circuit Board (PCB)-level optical interconnections. ${ }^{12}$ The out-of-plane coupler intends to turn the light path over $90^{\circ}$ by making use of a $45^{\circ}$ micro-mirror, to enable to couple surface-normal optoelectronic devices with board-embedded optical waveguides. Rather than using a standard $45^{\circ}$ micro-mirror, we present in this paper several enhancements to the coupler concept. To this end, we incorporate cylindrical micro-lenses for beam collimation, and the micro-mirror can be slightly curved as such that it not only reflects the beam by Total Internal Reflection (TIR), but includes some collimation functionality.

\footnotetext{
*E-mail: Jurgen.Van.Erps@vub.ac.be, Tel.: +32 247748 71, Fax.: +32 262934 50, http://tona.vub.ac.be/Tona/
} 
Optical simulations are performed to optimize the coupling efficiency of the out-of-plane coupler when used in a receiver $(\mathrm{Rx})$ configuration, i.e. when it is used to couple the light from the waveguides to a photodetector or a detector fiber. We use ASAP 2008 form Breault Research to perform non-sequential ray-tracing simulations to analyze the light transmission through the coupler. For the optimization of the curved micro-mirror shape, as well as the optimal cylindrical lens shape, we make use of Matlab optimization algorithms coupled to ASAP simulations. A tolerancing analysis for mechanical misalignments of the optimized out-of-plane coupler is also described.

We fabricate the optimal design of the out-of-plane couplers making use of our in-house rapid prototyping technology called Deep Proton Writing (DPW). The concept of DPW is based on a local irradiation of a PMMA polymer substrate with a pencil-like collimated proton beam according to a predefined pattern. The irradiation process changes the physical and chemical properties of the material in the irradiated zones. As a next step, a selective etching solvent is applied for the development of the irradiated regions, allowing us to achieve microoptical components with high quality optical sidewalls.

In a final section, we describe the characterization of the fabricated components. We make use of a non-contact optical surface profiler to measure the geometrical properties and surface roughness of the optical surfaces of the out-of-plane couplers, and we experimentally measure the optical coupling efficiency of the out-of-plane couplers when used in a fiber-to-fiber coupling scheme and when used on an optical PCB. A tolerance scan for mechanical misalignments is performed by making use a of sub-micron precision six-axis parallel kinematics (Hexapod) positioning robot. The experimentally measured optical performance is compared to the values expected from the optical simulations.

\section{DESIGN AND SIMULATIONS}

The starting point for our design is the discrete out-of-plane coupler with a standard $45^{\circ}$ micro-mirror we have recently introduced. ${ }^{12}$ We will optimize the out-of-plane coupling efficiency by replacing the $45^{\circ}$ micro-mirror with a curved mirror to combine TIR and collimation functionality, and by monolithically integrating a cylindrical micro-lens at the top facet to focus the light onto the detector. A schematical view of the enhanced out-of-plane coupler we propose here is shown in Fig. 1. For our simulations, the source is a Truemode multimode waveguide with a cross-section of $50 \times 50-\mu m^{2}$ and a numerical aperture (NA) of 0.3. The out-of-plane coupler is made out of PMMA polymer, which has a refractive index $n_{P M M A}=1.4804$ at the operating wavelength of 850- $n m$.

The design of the component comprises two steps. First, we will calculate the required curvature of the micromirror and the cylindrical top lens when an ideal point source is used. In a second step, we will approximate the calculated curves by a Bézier polynomial, which we will use as a starting point for an iterative optimization process which combines the non-sequential ray tracing capabilities of ASAP with the numerical algorithms of Matlab. ${ }^{13}$ In every iteration step of the optimization process, ASAP traces a set of rays through the system defined by a particular set of system parameters (Bézier points) calculated in Matlab. ASAP calculates the merit function, which is defined by the ray flux on the detector (within the desired directional acceptance distribution). This merit function is used by Matlab as feedback to calculate a new set of values for the system parameters. This is repeated until a minimum for the merit function is found. For the optimization part in Matlab, we use the multidimensional unconstrained non-linear minimization (downhill simplex) method, implemented in the fminsearch function. ${ }^{14}$

\subsection{Calculation of the starting values for an ideal point source}

To calculate the starting values, we assume that a point source $S$ is located at a distance $\Delta S=10-\mu m$ from the entrance facet, emitting $N$ rays with an angle $\theta$ with respect to the horizontal plane. We assign an NA of 0.3 to this point source, corresponding to the NA of a Truemode waveguide, by imposing that $-\sin ^{-1}(N A)<\theta<$ $\sin ^{-1}(N A)$. For the calculation of the mirror and lens curvatures, we make use of geometrical optics, which is a valid approach since the dimensions of the out-of-plane coupler are much larger than the wavelength at which it will be used (850-nm). 


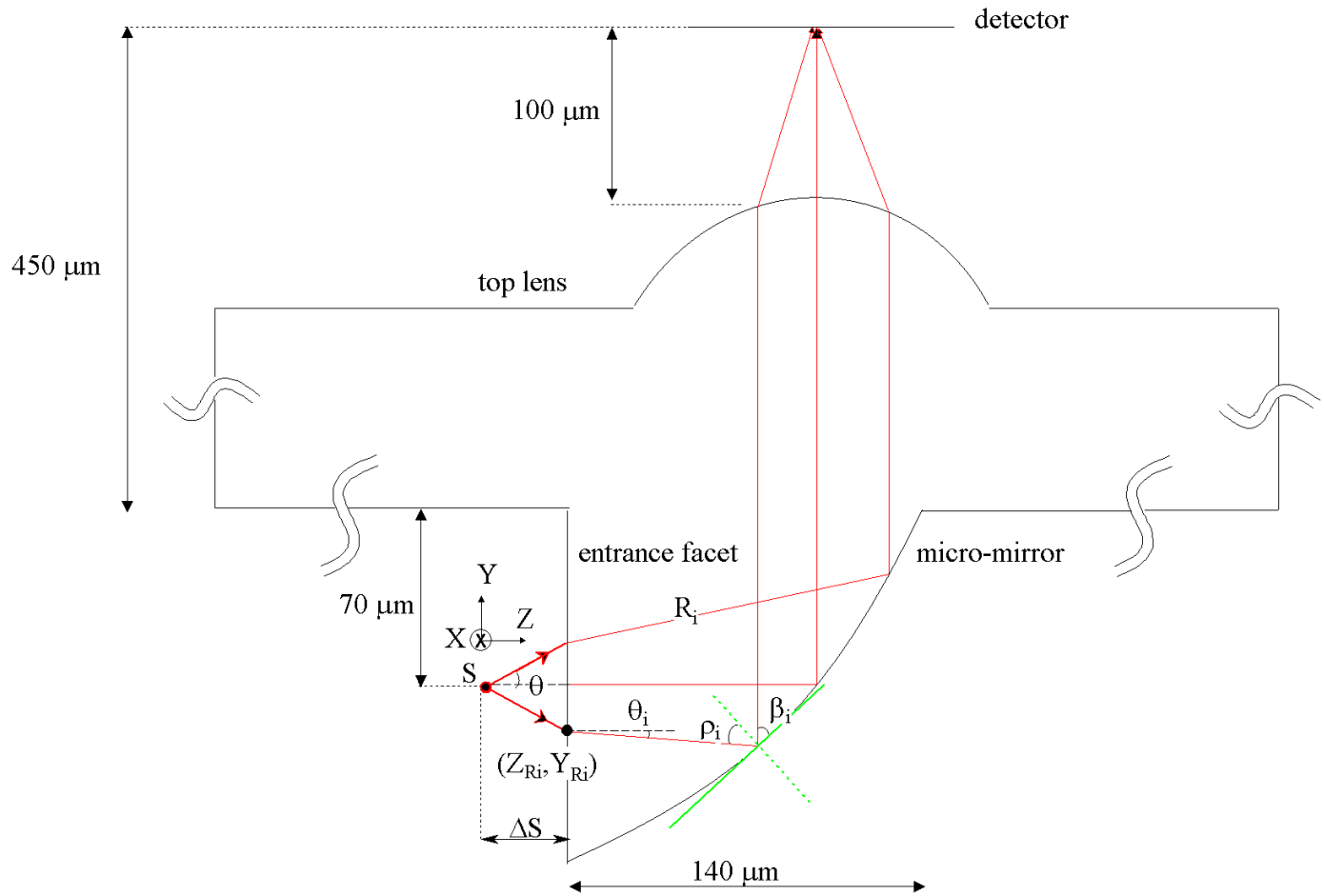

Figure 1. Schematic cross-section of the enhanced out-of-plane coupler, used at the receiver side with an ideal point source.

First, we determine the equation for each ray $R_{i}$ in the $\mathrm{YZ}$ plane using some simple trigonometry and the well-known law of Snell-Descartes at the air-PMMA interface at the entrance facet. This gives:

$$
y-y_{R_{i}}=\tan \left(\theta_{i}\right)\left(z-z_{R_{i}}\right)
$$

For a source with $\mathrm{NA}=0.3$, we know that $-11.67^{\circ}<\theta_{i}=\sin ^{-1}\left[\sin (\theta) / n_{P M M A}\right]<11.67^{\circ}$. Once we know the slope $\theta_{i}$ of each ray inside the coupler, we can determine the tangent to the micro-mirror curve required to reflect each rays upwards by TIR while ensuring beam collimation (i.e. reflection along the Y-axis) at the same time. These tangents will be used further on to interpolate the complete curve forming the micro-mirror. Recall that the condition for TIR is satisfied if the incidence angle of a ray is larger than the critical angle $\theta_{c}$, which equals $42.39^{\circ}$ for a PMMA-air interface. If we define $\beta$ as the angle between the tangent and the Y-axis, and $\rho_{i}$ as the incidence angle on the micro-mirror, as illustrated in Fig. 1, we see that $\rho_{i}=\beta_{i}-\theta_{i}$ and the condition for upward collimation $\left(\rho_{i}+\beta_{i}=90^{\circ}\right)$ can be expressed as follows:

$$
\begin{aligned}
& \beta_{i}=45^{\circ}+\theta_{i} / 2 \\
& \rho_{i}=45^{\circ}-\theta_{i} / 2
\end{aligned}
$$

Since we can write the condition for TIR as $\rho_{i}>\theta_{c}$, it is clear that it is impossible to impose TIR and upward collimation simultaneously for all rays. Indeed, TIR is not satisfied for $-11.67^{\circ}<\theta_{i}<-5.23^{\circ}$. This can be overcome by giving the entrance facet a slight tilt $\alpha$. Using Fig. 2 and the law of Snell-Descartes, we can write $\alpha$ as a function of $\theta$ and $\epsilon$ :

$$
\tan \alpha=\frac{\sin \theta-n_{P M M A} \sin \epsilon}{n_{P M M A} \cos \epsilon-\cos \theta}
$$

To ensure that $\theta_{i 2} \geq-5.23^{\circ}$, we need $\epsilon \leq-5.23^{\circ}$ which implies that the entrance facet angle should be chosen as $\alpha \geq 17.47^{\circ}$. We will check in ASAP if the gain made by introducing the facet tilt is not offset by larger backreflections at the entrance facet interface. As $\alpha$ remains fairly small, we expect the increase in backreflection, if any, to remain accordingly small. 


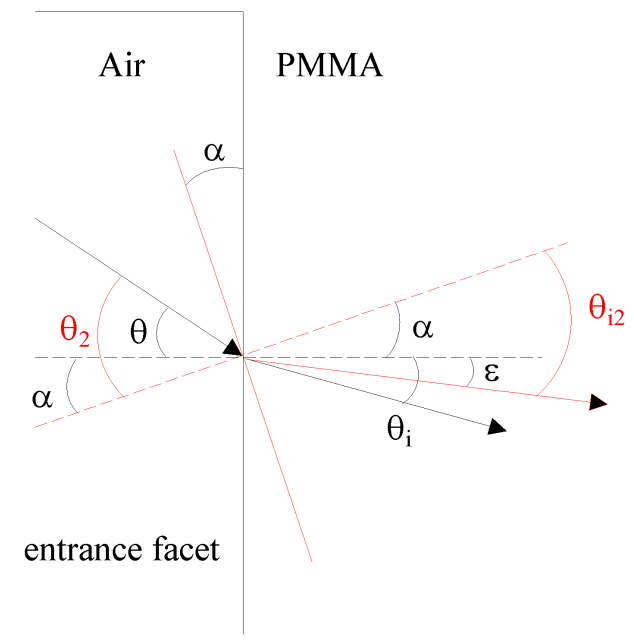

Figure 2. Introducing a tilt $\alpha$ of the entrance facet to ensure that all rays incident on the micro-mirror satisfy the TIR condition.

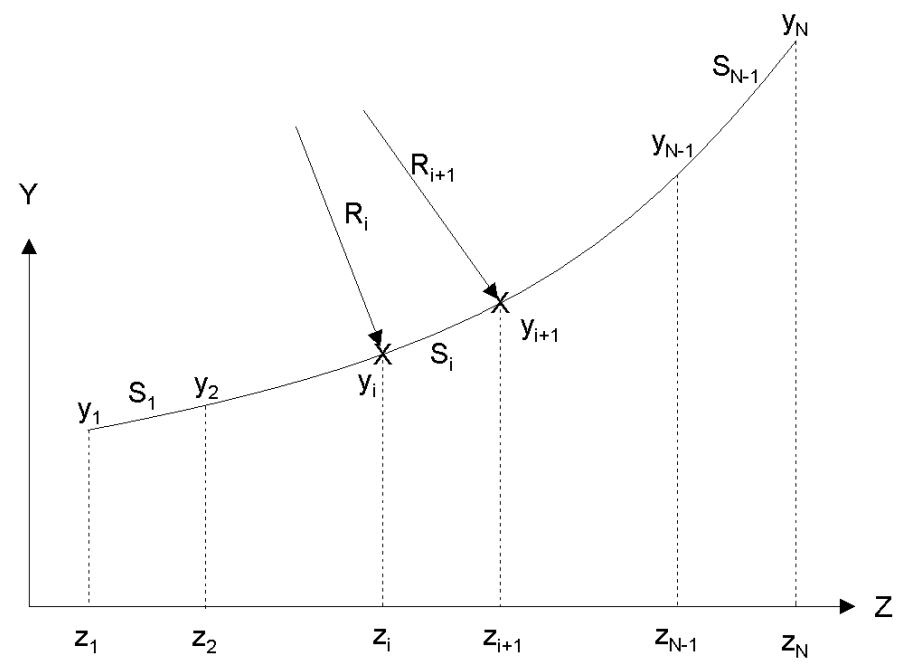

Figure 3. Quadratic interpolation of the micro-mirror curve.

\subsubsection{Quadratic interpolation of the micro-mirror curve}

From the above-calculated values of $\beta$ according to Eq. (2), we know the tangents to the micro-mirror curve in order to reflect each ray vertically (i.e. along the Y-axis). From here, we interpolate the required curve. We expect to end up with a curve that is close to a parabola, since it is well-known that all rays emitted by a point source placed at the focal point of a parabolic mirror will be collimated after reflection. We will however not end up with an exact parabola, since the rays refracted at the entrance facet do not originate from a common point (as opposed to the rays before refraction).

For the interpolation of the micro-mirror curve, we approximate the curve $y(z)$ as a consecutive series of quadratic polynomials $S_{i}(z)$, as illustrated in Fig. 3. For each interval $\left[z_{i}, z_{i+1}\right]$ we can define $S_{i}$ as follows:

$$
S_{i}(z)=a_{i}+b_{i}\left(z-z_{i}\right)+c_{i}\left(z-z_{i}\right)^{2}
$$

where $i=1,2 \ldots N-1$. In every point $z_{i}$, we impose:

$$
\begin{aligned}
& S_{i}\left(z_{i}\right)=a_{i}=y_{i} \\
& S_{i}^{\prime}\left(z_{i}\right)=b_{i}=y_{i}^{\prime}
\end{aligned}
$$


where ' denotes the first derivative. If we define $\Delta z_{i}=z_{i+1}-z_{i}$ and impose the continuity of $y^{\prime}(z)$, we get:

$$
c_{i}=\frac{1}{2 \Delta z_{i}}\left(y_{i+1}^{\prime}-y_{i}^{\prime}\right)
$$

If we furthermore impose continuity of $y(z)$, we get with Eqs. (6), (7) and (8):

$$
y_{i}+y_{i}^{\prime} \Delta z_{i}+\frac{1}{2 \Delta z_{i}}\left(y_{i+1}^{\prime}-y_{i}^{\prime}\right) \Delta z_{i}^{2}=y_{i+1}
$$

Eq. (9) can be rewritten as:

$$
\frac{y_{i+1}^{\prime}+y_{i}^{\prime}}{2}=\frac{y_{i+1}-y_{i}}{z_{i+1}-z_{i}}
$$

We know that every tangent $y_{i}^{\prime}$ to the mirror curve is associated with a ray $R_{i}$, so we can express that every point $\left(z_{i}, y_{i}\right)$ satisfies Eq. (10) as well as the equation for each ray $R_{i}$, given by Eq. (1). We can thus write, with $i=1,2 \ldots N-1$ :

$$
z_{i+1}\left[\tan \theta_{i+1}-\left(\frac{y_{i+1}^{\prime}+y_{i}^{\prime}}{2}\right)\right]-z_{i}\left[\tan \theta_{i}-\left(\frac{y_{i+1}^{\prime}+y_{i}^{\prime}}{2}\right)\right]=z_{R_{i+1}} \tan \theta_{i+1}-z_{R_{i}} \tan \theta_{i}-y_{R_{i+1}}+y_{R_{i}}
$$

Hence, we have $N-1$ equations versus $N$ variables. By assigning a value to $z_{0}$, which is the first point of our curved micro-mirror, all other points are defined.

\subsubsection{Converting the interpolated curve to a Bézier polynomial}

Although it is possible to import the curve found in the previous section directly into ASAP, we will first convert it to a Bézier curve to decrease the computing time of the simulation and, more importantly, to be able to define the geometry of our system with a limited number of variables (which can subsequently be optimized). We perform this conversion by means of a least squares approximation. A Bézier curve is defined as follows:

$$
\vec{B}(t)=\sum_{i=0}^{n} \frac{n !}{i !(n-i) !} \vec{P}_{i}(1-t)^{n-i} t^{i}
$$

where $n$ is the order of the Bézier curve, $t \in[0,1], \overrightarrow{P_{0}}$ and $\overrightarrow{P_{n}}$ are respectively the starting point and the end point of the Bézier curve, and $\vec{P}_{1} \ldots \vec{P}_{n-1}$ are called the control points of the curve.

To be able to perform a least squares approximation, we should know the value of $t$ at each point on the Bézier curve. To this end, we approximate $t$ as follows:

$$
t_{j}=\frac{\sum_{i=1}^{j-1} \sqrt{\left(z_{i+1}-z_{i}\right)^{2}+\left(y_{i+1}-y_{i}\right)^{2}}}{\sum_{i=1}^{N-1} \sqrt{\left(z_{i+1}-z_{i}\right)^{2}+\left(y_{i+1}-y_{i}\right)^{2}}}
$$

with $j=2,3 \ldots N$ where $N$ is the number of points on the interpolated curve and $t_{1}=0$. We can now find the coefficients $\overrightarrow{P_{0}}, \overrightarrow{P_{1}} \ldots \vec{P}_{n}$ that are in best accordance with the following set of linear equations:

$$
\begin{array}{r}
A_{i j} \vec{P}_{j}^{(z)}=\vec{z}_{i} \\
A_{i j}=\frac{n !}{j !(n-j) !}\left(1-t_{i}\right)^{n-j} t_{i}^{j}
\end{array}
$$

with $i=1,2 \ldots N$ and $j=0,1 \ldots n$. From here, the vector with the $z$-coordinates of the Bézier curve can be calculated as $\vec{P}^{(z)}=\left(A^{T} A\right)^{-1} A^{T} \vec{z}$, where $A^{T}$ denotes the transpose of matrix $A$. And, in a completely analog way, $\vec{P}^{(y)}=\left(A^{T} A\right)^{-1} A^{T} \vec{y}$. As expected, the micro-mirror curve can be well approximated by a second-order Bézier curve, since its shape is close to a parabola. 


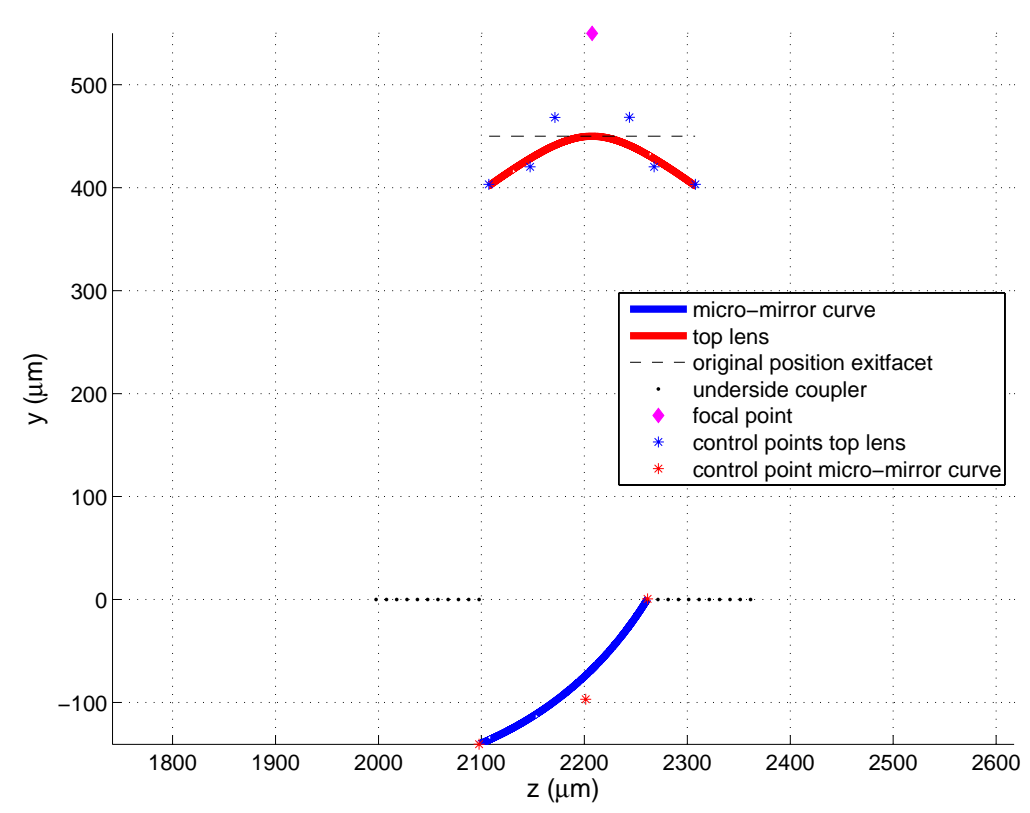

Figure 4. Resulting Bézier curves for the micro-mirror and the top lens for an entrance facet angle $\alpha=17^{\circ}$.

\subsubsection{Calculation of the cylindrical lens curve at the top facet}

After calculating the micro-mirror curve to ensure beam collimation after reflection, we can calculate the shape of the cylindrical lens at the top facet to focus the light on the detector in a similar manner. We choose the lens' focal length equal to 100- $\mu \mathrm{m}$. Since the shape of the top lens is a hyperbola, we use a fifth-order Bézier to approximate the curve. The resulting Bézier curves for the micro-mirror and the top lens are shown in Fig. 4.

\subsection{Iterative ASAP-Matlab system optimization}

Now that we have the determined the Bézier curves, we are ready to define the system geometry in the nonsequential ray tracing software ASAP by means of a set of Bézier control points. Notice that for all simulations described here, we didn't take the scattering due to surface roughness into account, but we did incorporate Fresnel losses occuring at the different interfaces. We start with investigating the influence of tilting the entrance facet. To this end, we define three detectors in our simulation, as shown in Fig. 5(a). This figure shows a ray tracing through the system with the calculated curved mirror, but with an untilted entrance facet (i.e. $\alpha=0^{\circ}$ ), for an ideal point source. The detector 1 is the actual detector on which we want to maximize the flux. Detector 2 is placed behind the micro-mirror and measures the amount of light that is lost by rays that do not satisfy the condition for TIR. Detector 3 measures the amount of light that is backreflected at the entrance facet interface. In these first simulations with a point source, the size of detector 1 is $250 \times 250-\mu m^{2}$. In Fig. 5 (b) and (c), the entrance angle $\alpha$ is changed to respectively $10^{\circ}$ and $17^{\circ}$. A comparison between these three configurations is shown in Table 1. This table shows that introducing an entrance facet tilt $\alpha$ increases the efficiency of the system by decreasing the TIR loss at the micro-mirror. However, as we shift from $\alpha=10^{\circ}$ to $\alpha=17^{\circ}$, all rays satisfy the TIR condition at the micro-mirror, but no increase of the efficiency on detector 1 is observed. Therefore, we decide to fix the entrance facet angle to $\alpha=10^{\circ}$ for the rest of our optimization process.

As a next step, we make the transition from a point source to a Truemode waveguide source and we choose the size of detector 1 as $100 \times 100-\mu m^{2}$. A ray tracing through that system is shown in Fig. 6(a). The coupling efficiency of the system has now dropped to $20.1 \%$, as can be seen in Table 2 . We now start the optimization procedure described earlier, in order to optimize the mirror curvature. The resulting system geometry after this first optimization step is shown in Fig. 6(b) and shows an efficiency of $55.7 \%$. Finally, we introduce the cylindrical top lens in the system and run the second optimization process. This increases the system efficiency further to $58.2 \%$ and the resulting optimal system geometry is shown in Fig. 7(b). This is the system we will fabricate, as will be discussed in the following section. For comparison purposes, Fig. 7(a) shows a ray trace 


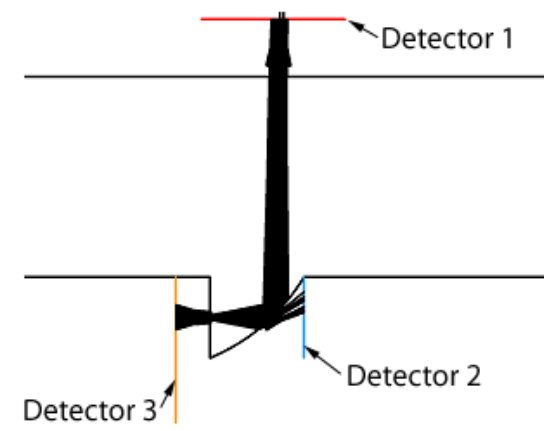

(a)

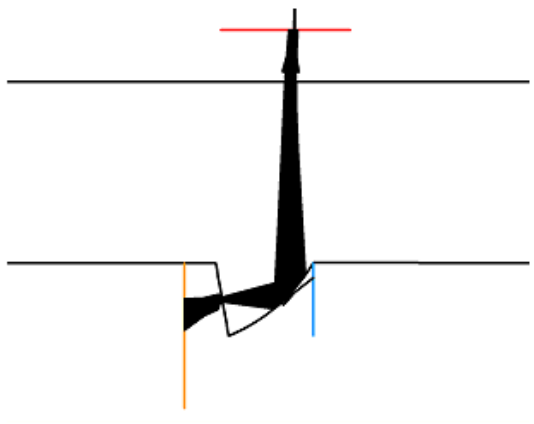

(b)

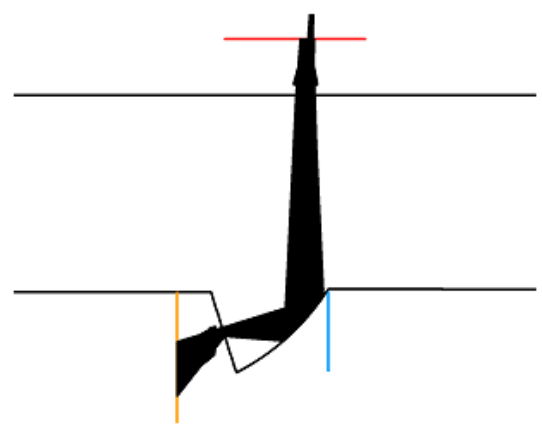

(c)

Figure 5. System configurations to determine the influence of the tilt of the entrance angle: $\alpha=0^{\circ}$ (a), $\alpha=10^{\circ}$ (b), $\alpha=17^{\circ}$ (c). A point source was used for the ray tracing.

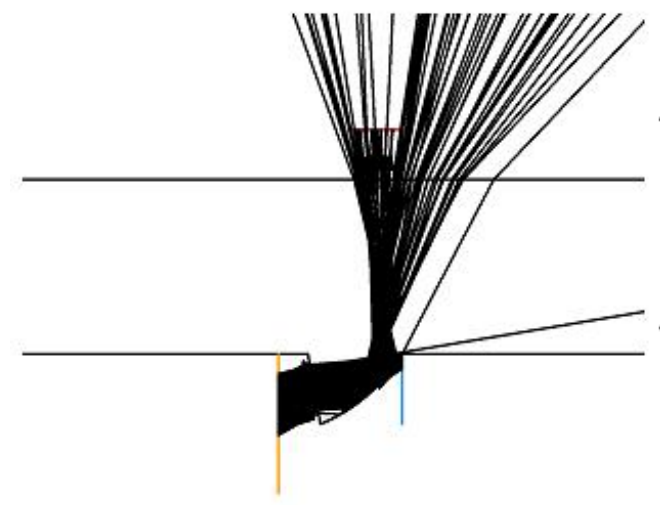

(a)

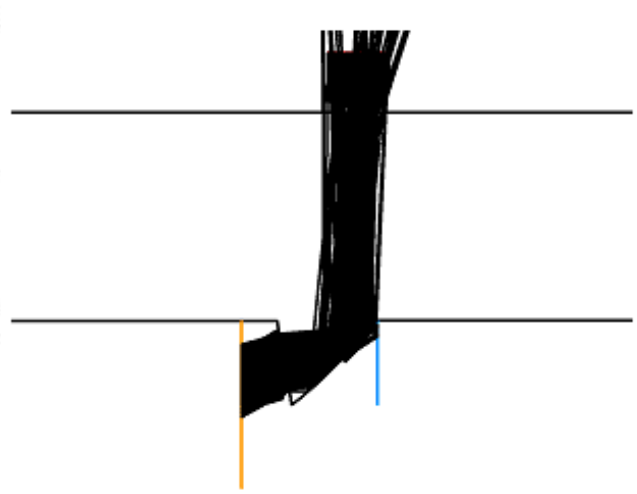

(b)

Figure 6. Transition from a point source to a Truemode waveguide source (a), and the resulting system geometry after optimization of micro-mirror curvature of the out-of-plane coupler (b).

through the system geometry we obtained from our calculations in the previous sections, when using a point source.

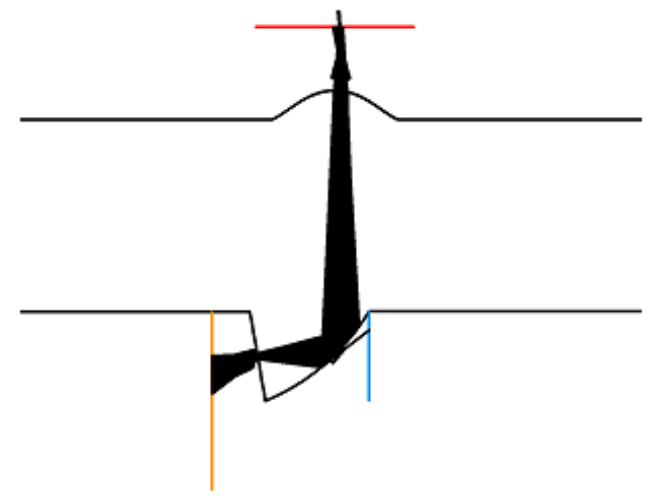

(a)

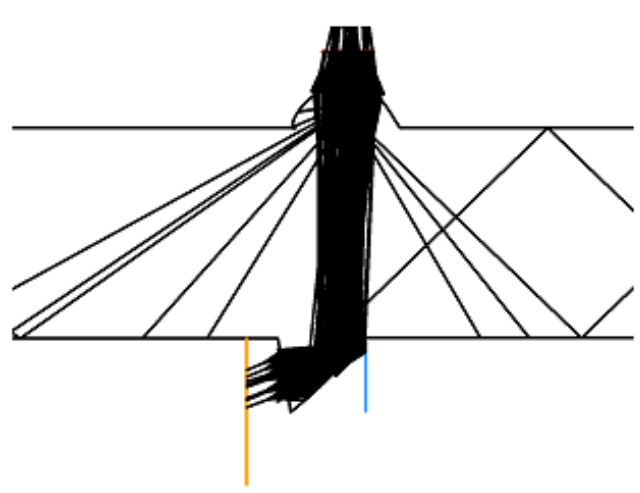

(b)

Figure 7. Introduction of the cylindrical top lens in the out-of-plane coupler for a point source (a), and the resulting system geometry after optimization of the cylindrical top lens for a Truemode waveguide source (b). 
Table 1. ASAP simulation results showing the influence of the entrance facet angle for a point source. The table shows the efficiency measured on the three detectors defined in Fig. 5 (a). The size of detector 1 is $250 \times 250$ - $\mu \mathrm{m}^{2}$ for these simulations.

\begin{tabular}{|c|c||c|c|c|}
\hline System & $\alpha$ & Detector 1 & Detector 2 & Detector 3 \\
\hline \hline Fig. 5(a) & $0^{\circ}$ & $79.6 \%$ & $8.7 \%$ & $5.8 \%$ \\
Fig. 5(b) & $10^{\circ}$ & $85.4 \%$ & $1.6 \%$ & $6.2 \%$ \\
Fig. 5(c) & $17^{\circ}$ & $85.4 \%$ & $0.0 \%$ & $6.3 \%$ \\
\hline
\end{tabular}

Table 2. Results of the ASAP-Matlab optimization process for a Truemode waveguide source. The table shows the efficiency measured on the three detectors defined in Fig. 5(a). The size of detector 1 is $100 \times 100-\mu m^{2}$ for this optimization, and the entrance facet angle is fixed at $\alpha=10^{\circ}$.

\begin{tabular}{|c|c||c|c|c|}
\hline System & Optimization state & Detector 1 & Detector 2 & Detector 3 \\
\hline \hline Fig. 6(a) & Unoptimized & $20.1 \%$ & $15.9 \%$ & $4.4 \%$ \\
Fig. 6(b) & Mirror curvature optimized & $55.7 \%$ & $2.4 \%$ & $5.9 \%$ \\
Fig. 7(b) & Mirror and top lens optimized & $58.2 \%$ & $2.4 \%$ & $4.5 \%$ \\
\hline
\end{tabular}

\section{FABRICATION THROUGH DEEP PROTON WRITING}

For the manufacturing of the pluggable out-of-plane coupling components, we use the rapid prototyping technology of Deep Proton Writing (DPW). ${ }^{15}$ It consists of a patterned 8.3- $\mathrm{MeV}$ proton irradiation of 500- $\mu \mathrm{m}$ thick polymer photoresist (PMMA), followed by a selective etching process to remove the exposed zones. The sample is moved according to a predefined pattern in steps of 500- $\mathrm{nm}$ with an accuracy of 50- $\mathrm{nm}$, while depositing $2.4 \times 10^{4} / \mu m^{2}$ protons per step to achieve the highest quality optical surfaces. Typical proton currents for the $140-\mu m$ proton beam diameter are 200- $p A$, resulting in total processing times of about 5 hours (development included) for a component with dimensions of 4.5- $\mathrm{mm} \times 0.5-\mathrm{mm} \times 0.5-\mathrm{mm}$. Fig. 8 shows the prototyped component. Due to the finite beam size of $140-\mu m$, there is some rounding at the corners of the component, but this does not affect its optical functionality.

A non-contact optical surface profiler (WYKO NT-2000, Veeco) was used to characterize the surface roughness of the optical sidewalls of the components. The RMS roughness $R_{q}$ was measured by averaging 5 measurements of an area of $58 \times 46-\mu m^{2}$ at random positions, resulting in $R_{q}=12.2-n m \pm 3.5-n m$. The peak-to-valley flatness $R_{t}$ of DPW surfaces is measured to be as small as $R_{t}=4.0-\mu m$ over the total component thickness of $500-\mu \mathrm{m}$. This is due to the proton scattering during the interaction with the PMMA. ${ }^{15} \mathrm{We}$ can conclude that the developed DPW surfaces have a very high quality: almost flat and with a very low RMS surface roughness. However, the ultimate quality assessment of the fabricated out-of-plane coupler is the experimental measurement of the coupling efficiency that can be achieved, which will be discussed in the next section.

It is obvious that DPW is not a mass fabrication technique as such. However, one of its assets is that once the master component has been prototyped, a metal mould can be generated from the DPW master by applying electroplating. After removal of the plastic master, this metal mould can be used as a shim in a final microinjection moulding or hot embossing step. ${ }^{16,17}$ This way, the master component can be mass-produced at low
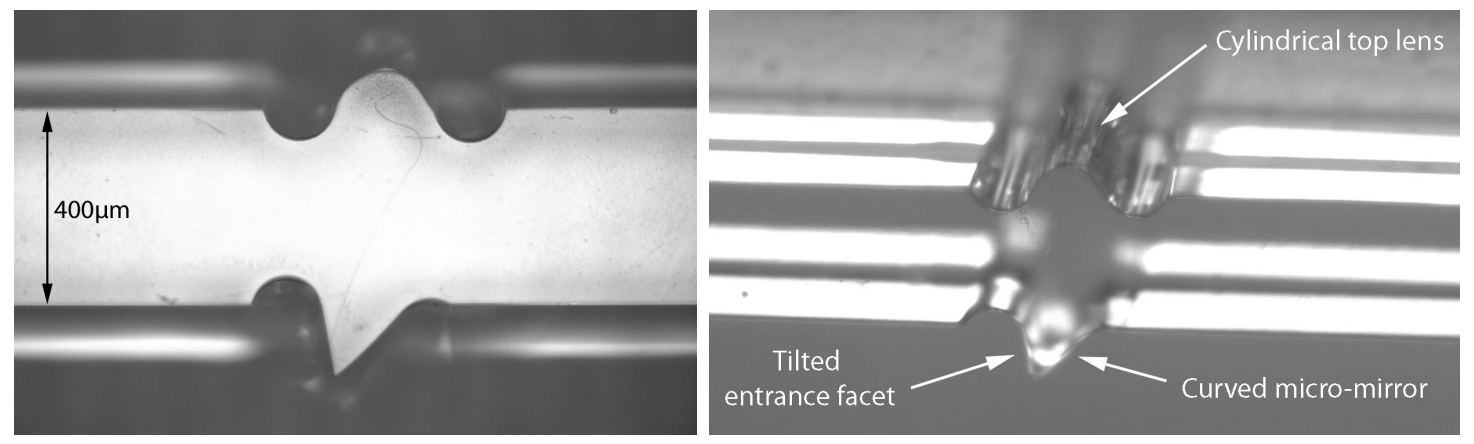

Figure 8. Pictures of the fabricated enhanced out-of-plane coupler using Deep Proton Writing. 


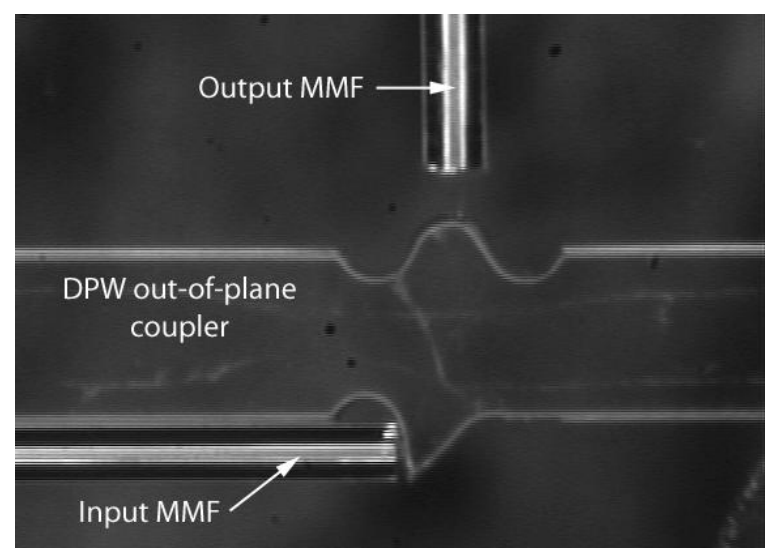

Figure 9. Experimental setup for the fiber-to-fiber coupling efficiency measurements of the enhanced out-of-plane coupler. Table 3. Out-of-plane coupling efficiency measurement results for the coupler with a standard $45^{\circ}$ micro-mirror, and for the enhanced coupler with curved micro-mirror and integrated cylindrical lens at the top facet.

\begin{tabular}{|c||c|c|}
\hline Component & Fiber-to-fiber & On PCB demonstrator \\
\hline \hline Standard coupler & $83.7 \%(-0.77-d B)$ & $27.1 \%(-5.67-d B)$ \\
Enhanced coupler & $80.0 \%(-0.97-d B)$ & $33.9 \%(-4.70-d B)$ \\
\hline
\end{tabular}

cost in a wide variety of high-tech plastics.

\section{EXPERIMENTS}

To measure the coupling efficiency that can be achieved with the DPW enhanced out-of-plane coupler, we perform the same experiments as for the out-of-plane coupler with a standard $45^{\circ}$ micro-mirror. ${ }^{12}$ We first characterize the component in a fiber-to-fiber coupling scheme. Here, the input fiber is a multimode silica fiber (MMF) with a core diameter of $50-\mu m$ and $\mathrm{NA}=0.2$, and the output fiber is a silica MMF with $100-\mu m$ core and NA=0.29, mounted on a Hexapod six-axis parallel kinematics motion robot. This allows us to both position the fiber with an accuracy of 300- $\mathrm{nm}$ and to perform two-axis scans to measure the tolerance for mechanical misalignments of the detector fiber. The reference measurement consists of an in-line butt coupling of both fibers.

In a second test, we plug the component into a PCB with integrated multimode optical waveguides. The Truemode Backplane ${ }^{\mathrm{TM}}$ polymer waveguides have a cross-section of $50 \times 50-\mu \mathrm{m}^{2}$ and an NA of 0.3 . A detailed description of the waveguide fabrication and characterization was published earlier. ${ }^{18}$ The micro-cavity that accomodates the DPW out-of-plane coupler was created using laser ablation. For the fabrication of the microcavity, a KrF excimer laser is tilted by $6^{\circ}$ such that a vertical end-facet of the waveguides can be created. ${ }^{19}$ The DPW out-of-plane coupler is inserted manually in the cavity by means of a tweezer. The measured loss in both schemes is mentioned in Table 3. The loss on the PCB demonstrator includes the butt coupling from the input fiber to the PCB-integrated waveguide, the propagation in the waveguide over a length of about 5- $\mathrm{cm}$, the coupling towards the out-of-plane coupler and from the coupler to the detector fiber. No index matching gel was used at any point. We know that the propagation loss of the PCB-integrated waveguides is $0.13-\mathrm{dB} / \mathrm{cm}$ and the average in-plane coupling loss is $1.77-d B .{ }^{18}$ Hence the coupling loss can be estimated to be $-3.27-d B$ for the standard coupler and $-2.30-d B$ for the enhanced coupler. This means that in this configuration, a coupling loss improvement of about 1- $d B$ was achieved by introducing the mirror and top lens curvature in the coupler. However, the measured efficiency of about $34 \%$ is much lower than the simulated efficiency of the optimal system $(58.2 \%)$. This discrepancy can be due to the fact that no scattering loss was taken into account for the simulations and since the enhanced out-of-plane coupler was inserted manually in the PCB cavity, misalignments might occur in the experimental setup.

The slight decrease in coupling efficiency for the fiber-to-fiber coupling scheme is due to the fact that the collimation/focussing only occurs in one direction. If the detector is placed in the focal point of the top lens, the larger optical path length apparently leads to a decrease in efficiency. Moreover, it should be noted that the 

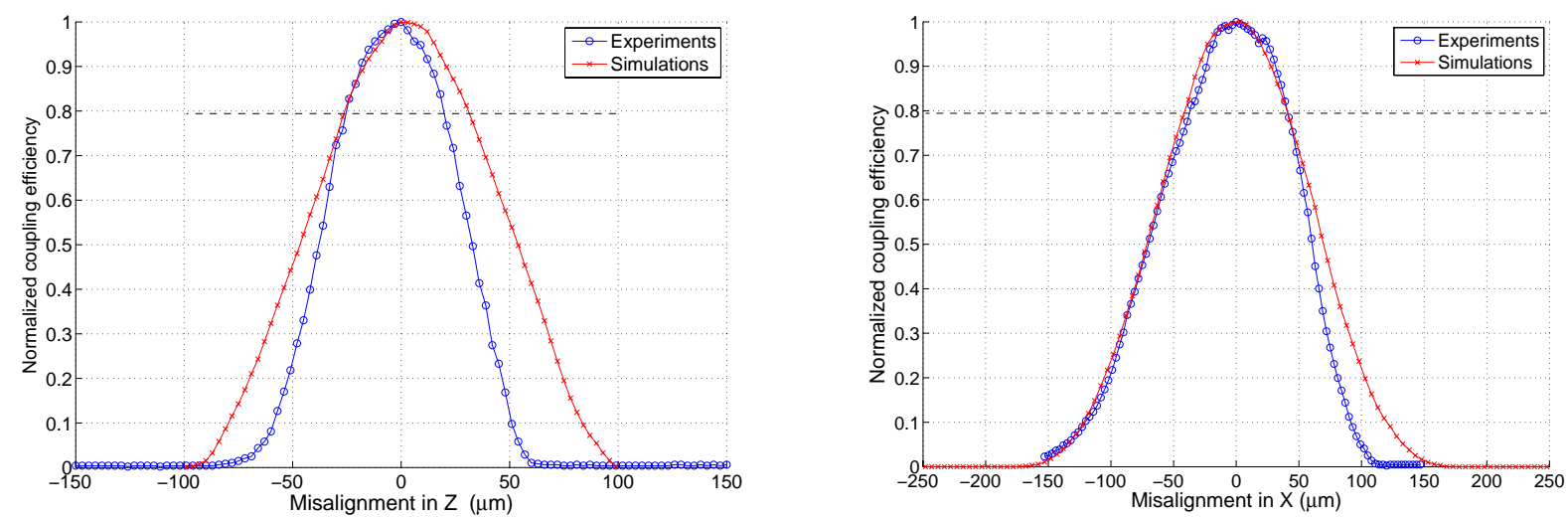

Figure 10. Simulated versus measured MMF detector misalignment tolerance for the enhanced out-of-plane coupler on the PCB demonstrator.

Table 4. Measured versus simulated -1- $d B$ MMF detector alignment tolerance range for the enhanced out-of-plane coupler when used on a PCB.

\begin{tabular}{|c||c|c|}
\hline & Along X & Along Z \\
\hline \hline Simulations & $\pm 40-\mu m$ & $\pm 20-\mu m$ \\
Experiments & $\pm 40-\mu m$ & $\pm 25-\mu m$ \\
\hline
\end{tabular}

enhanced out-of-plane coupler was designed for a Truemode waveguide source, with an NA of 0.3 , and not for an MMF source with an NA of 0.2 .

Finally, we performed a measurement of the MMF detector misalignment to determine the $-1-d B$ alignment tolerance range. The results are shown in Fig. 10 and in Table 4. A good correspondence between the measured and the simulated tolerance can be observed. The tolerance scans also clearly show the focusing of the light in one direction (along Z). To further enhance the coupling efficiency, we are currently investigating the use of a $2 \mathrm{D}$ curvature of the micro-mirror and of the top facet lens.

\section{CONCLUSION}

We have shown the design and simulation of an enhanced out-of-plane coupling component for PCB-level optical interconnections, incorporating a curved micro-mirror and a cylindrical micro-lens. The design was optimized by combining the optical simulation software ASAP and the downhill simplex numerical optimization algorithm as it is implemented in Matlab. We have shown that we are able to fabricate a high-quality prototype using Deep Proton Writing, which is compatible with replication technologies, paving the way towards mass-fabrication at low cost in a wide variety of high-tech plastics.

The discrete out-of-plane couplers are very versatile, since they can be readily inserted into cavities formed in PCB-integrated optical waveguides. In this configuration, a coupling efficiency improvement of about 1- $d B$ was measured in comparison with an out-of-plane coupler with a standard $45^{\circ}$ micro-mirror. This can be further improved by introducing curvatures in two dimensions and/or by applying a metal reflection coating on the micro-mirror.

\section{ACKNOWLEDGMENTS}

This work was supported in part by DWTC-IAP6, FWO, IWT, the European Network of Excellence on MicroOptics NEMO and the OZR of the Vrije Universiteit Brussel. The work of J. Van Erps and C. Debaes was supported by the FWO (Fund for Scientific Research - Flanders) under a research fellowship. N. Hendrickx was financially supported by the Flemish IWT (Institute for the Promotion of Innovation by Science and Technology). 


\section{REFERENCES}

[1] D.A.B. Miller, "Rationale and challenges for optical interconnects to electronic chips," Proc. IEEE, Vol. 88, No. 6, pp. 728-749, 2000.

[2] M.W. Haney, H. Thienpont and T. Yoshimura, "Introduction to the issue on optical interconnects," IEEE J. Select. Topics Quantum Electron., Special Issue on Optical Interconnects, Vol. 9, No. 2, pp. 347-349, 2003.

[3] R.T. Chen et al., "Fully embedded board-level guided-wave optoelectronic interconnects," Proc. IEEE, Vol. 88, No. 6, pp. 780-793, 2000.

[4] S. Uhlig and M. Robertsson, "Limitations to and solutions for optical loss in optical backplanes," J. Lightwave Technol., Vol. 24, No. 4, pp. 1710-1724, 2006.

[5] R. Yoshimura et al., "Polymeric Optical Waveguide Films with $45^{\circ}$ Mirrors Formed with a $90^{\circ}$ V-Shaped Diamond Blade," Electron. Lett., Vol. 33, No. 15, pp. 1311-1312, 1997.

[6] A. Glebov, J. Roman, M.G. Lee and K. Yokouchi, "Optical Interconnect Modules with Fully Intgrated Reflector Mirrors," IEEE Photon. Technol. Lett., Vol. 17, No. 7, pp. 1540-1542, 2005.

[7] Y. Liu, L. Lin, C. Choi, B. Bihari and R.T. Chen, "Optoelectronic Integration of Polymer Waveguide Array and Metal-Semiconductor-Metal Photodetector Through Micromirror Couplers," IEEE Photon. Technol. Lett., Vol. 13, No. 4, pp. 355-257, 2001.

[8] M. Immonen, W. Jianbo and J. Kivilathi, "Fabrication of polymer optical waveguides with integrated micromirrors for out-of-plane surface normal optical interconnects," Proc. 4th IEEE Int. Conf. Polymers and Adhesives in Microelectronics and Photonics - Polytronic2004, pp. 206-210, 2004.

[9] J.-S. Kim and J.-J. Kim, "Fabrication of Multimode Polymeric Waveguides and Micro-mirrors using Deep X-ray Lithography," IEEE Photon. Technol. Lett., Vol. 16, No. 3, pp. 798-800, 2004.

[10] G. Van Steenberge et al., "MT-Compatible Laser-Ablated Interconnections for Optical Printed Circuit Boards," J. Lightwave Technol., Vol. 22, No. 9, pp. 2083-2090, 2004.

[11] N. Hendrickx, J. Van Erps, G. Van Steenberge, H. Thienpont and P. Van Daele, "Laser Ablated Micromirrors for Printed Circuit Board Integrated Optical Interconnections," IEEE Photon. Technol. Lett., Vol. 19, No. 11, pp. 822-824, 2007.

[12] J. Van Erps, N. Hendrickx, C. Debaes, P. Van Daele and H. Thienpont, "Discrete Out-of-Plane Coupling Components for Printed Circuit Board-Level Optical Interconnections," IEEE Photon. Technol. Lett., Vol. 19, No. 21, pp. 1753-1755, 2007.

[13] B. Van Giel, Y. Meuret and H. Thienpont, "Using a fly's eye integrator in efficient illumination engines with multiple light-emitting diode light sources," Opt. Eng., Vol. 46, No. 4, 043001, 2007.

[14] W. Press, S.A. Teulolsky, W.T. Vetterling and B. Flannery, Numerical recipes in C: The art of scientific computing (Cambridge University Press, 1992).

[15] C. Debaes et al., "Deep Proton Writing: a rapid protoyping polymer microfabrication tool for micro-optical modules," New J. Phys., Vol. 8, pp. 270-288, 2006.

[16] M. Heckele and W.K. Schomburg, "Review on micro molding of thermoplastic polymers," J. Micromech. Microeng., Vol.14, pp. R1-R14, 2004.

[17] J. Van Erps et al., "Replication of deep micro-optical components prototyped by Deep Proton Writing," Proc. SPIE, Vol. 6992, Paper No. 6992-8, 2008.

[18] G. Van Steenberge et al., "Laser Ablation of Parallel Optical Interconnect Waveguides," IEEE Photon. Technol. Lett., vol. 18, no. 9, pp. 1106-1108, 2006.

[19] J. Van Erps et al., "Deep lithography with protons to prototype pluggable micro-optical out-of-plane coupling structuresfor multimode waveguides," Proc. SPIE, Vol. 5956, pp. 52-63, 2005. 